\title{
Malignant Neoplasm of Multiple Primary Sites
}

National Cancer Institute

\section{Source}

National Cancer Institute. Malignant Neoplasm of Multiple Primary Sites. NCI Thesaurus.

Code C35427.

A malignant neoplasm arising in multiple primary sites. 